\title{
Génération de rayonnement VUV et XUV cohérent par conversion de fréquence dans les gaz et les vapeurs métalliques. Utilisation en physique atomique et moléculaire
}

\section{J. L. Lemaire}

Observatoire de Paris, DAMAp \& URA 812 du CNRS, 5 Place J. Janssen, 92195 Meudon Cedex, France

\& Université de Cergy-Pontoise, France

\begin{abstract}
Frequency upconversion of high-power visible or ultraviolet laser radiation by nonlinear optical processes in gases and metal vapors is a well established method to build coherent, high spectral brightness and tunable vacuum or extreme ultraviolet sources ("VUV" or "XUV" lasers). They provide a powerful tool for spectroscopy of atoms and small molecules through direct excitation of high lying electronic states such as Rydberg or valence states. Fundamental applications concern high resolution spectroscopy, molecular structure and molecular dynamics in the process of photodissociation or photoionization. Application fields are trace detection, plasma diagnostics, surface photochemistry.
\end{abstract}

\section{Introduction}

Les lasers sont l'instrument de choix pour les études de base de spectroscopie atomique et moléculaire et leur extension à toute une variété de problèmes de photophysique et de physicochimie moléculaire. On dispose aujourd'hui d'une grande variété de sources lasers directes fournissant un rayonnement intense, monochromatique et accordable, de l'infrarouge à l'ultraviolet $(1,2 \mu \mathrm{m}$ à $190 \mathrm{~nm})$. Cependant, la nécessité apparait dans un grand nombre de cas, d'accéder à une excitation par un rayonnement dans l'ultraviolet du vide $(\lambda<185 \mathrm{~nm}$ ou $E>6,7 \mathrm{eV})$. En effet, outre les atomes, de nombreuses molécules légères, qui jouent un rôle d'une grande importance dans différents champs de la physique (astrophysique des milieux interstellaires, planétaires ou cométaires, physique de l'atmosphère et de la stratosphère) telles $\mathrm{H}_{2}, \mathrm{~N}_{2}, \mathrm{O}_{2}, \mathrm{CO}, \mathrm{CO}_{2} \ldots$ ont leur plus bas niveau d'énergie électronique excité, situé dans cette gamme d'énergie. Ainsi, le besoin croissant d'études spectroscopiques ou photophysiques de telles espèces atomiques ou moléculaires, utilisant l'excitation de niveaux électroniques élevés ou d'états de Rydberg, a incité au développement concommitant de sources cohérentes dans l'ultraviolet du vide. L'utilisation de telles sources, de préférence à une excitation multiphotonique de même énergie totale, simplifie considérablement, dans bien des cas, l'interprétation des résultats spectroscopiques. Ces sources fondées sur les processus de l'optique non linéaire sont utilisées, soit indépendamment (technique de fluorescence induite par laser...), soit en combinaison avec une ou plusieurs sources laser visibles (technique de pompage et de sondage par exemple). Deux sources VUV peuvent parfois être nécessaires, la première réalisant la photodissociation de la molécule étudiée et la seconde sondant les fragments produits. Notons enfin que ce rayonnement peut être 
considéré sous son aspect "source primaire d'excitation", mais également comme outil de diagnostic du milieu dans lequel intervient le processus non linéaire.

\section{Généralités}

A quelques exceptions près, il n'existe pas aujourd'hui de laser produisant directement (par le mécanisme habituel d'inversion de population entre états électroniques du milieu actif) un rayonnement intense, monochromatique et accordable dans l'ultraviolet du vide. Les lasers fonctionnant dans cette gamme de longueurs d'onde émettent en général un rayonnement à large bande autour de transitions discrètes (excimères de gaz rare $\mathrm{Kr}$, Xe ou $\mathrm{Ar}$, exciplexes de gaz rares et d'halogènes XeCL, ArF ou gaz moléculaires $\mathrm{H}_{2}, \mathrm{CO}, \mathrm{F}_{2}$ ) [1].

Une large gamme d'accordabilité, qui est l'une des nécessités premières, avec la finesse spectrale, de la spectroscopie ne pourra être obtenue que par les méthodes indirectes de conversion de fréquence offertes par l'optique non linéaire. L'utilisation des processus de l'optique non linéaire dans un milieu transparent est aujourd'hui une méthode bien établie pour construire des sources lumineuses dans les gammes spectrales de l'ultraviolet du vide (VUV: $105 \mathrm{~nm}<\lambda<190 \mathrm{~nm}$ ) ou de l'extrême ultraviolet (XUV: $\lambda<105 \mathrm{~nm}$ ). Depuis les travaux de deux groupes de pionniers [2],[3], les champs théoriques et expérimentaux des processus de l'optique non linéaire ont été largement explorés, démontrant que la génération d'harmonique ou le mélange de fréquence par combinaison d'une ou plusieurs sources-laser visibles de forte puissance produisait un rayonnement cohérent accordable et de forte brillance spectrale désigné sous les termes de "laser VUV ou XUV". Les caractéristiques finales de la source (monochromaticité et accordabilité) résulteront en grande partie des caractéristiques des sources génératrices.

De nombreux ouvrages traitent des processus de l'optique non linéaire [4]. Le principe en est très succinctement le suivant : le champ électrique total $\mathscr{E}$ de l'onde lumineuse traversant un milieu actif crée une polarisation induite de ce milieu qui peut s'écrire sous la forme d'un développement en série $\mathrm{P}=\Sigma \chi(\mathrm{i}) \mathscr{E}^{\mathrm{i}}$ faisant intervenir les termes $\chi(\mathrm{i})$ d'ordre $\mathrm{i}$ du tenseur de susceptibilité dipolaire. Les termes non linéaires d'ordre élevés apparaîtront lorsque l'amplitude du champ électrique augmentera. Les propriétés de symétrie du milieu actif déterminent les ordres pouvant apparaître. Ainsi, dans le cas des cristaux anisotropiques usuels, c'est le terme d'ordre 2 qui est actif, donnant naissance au doublement ou au mixage de fréquence, technique couramment utilisée dans les lasers. Cependant, les cristaux sont opaques en deçà de $195 \mathrm{~nm}$ (limite d'utilisation du $\beta$-borate de Barium $\mathrm{BBaB}_{2} \mathrm{O}_{4}$ ou $\mathrm{BBO}$ ). Cette limite pourrait être repoussée à $165 \mathrm{~nm}$ avec un nouveau cristal en cours d'étude [5] (tri-borate de Lithium $\mathrm{LiB}_{3} \mathrm{O}_{5}$ ou $\mathrm{LBO}$ ).

Pour l'instant, avant $195 \mathrm{~nm}$, les seuls milieux de conversion utilisables sont les gaz rares, les vapeurs métalliques ou certains gaz moléculaires pour lesquels n'apparaissent que les termes d'ordre impair. Le terme de troisième ordre donne naissance au triplement de fréquence THG (third harmonic generation) dans le cas d'un seul laser excitateur. Le THG est un cas particulier du mélange, à quatre ondes FWM (four wave mixing) dans le cas de 2 ou 3 lasers sources. On distingue deux processus de FWM, le mélange additif SFM dans lequel les trois fréquences des lasers sources s'ajoutent pour donner $\omega_{V U V}=\omega_{1}+\omega_{2}+\omega_{3}$ (ou $\omega_{V U V}=2 \omega_{1}+\omega_{3}$ ) et le mélange soustractif DFM pour lequel $\omega_{V U V}=\omega_{1}+\omega_{2}-\omega_{3}\left(\right.$ ou $\left.\omega_{V U V}=2 \omega_{1}-\omega_{3}\right)$. Des termes d'amplification paramétrique sont également très facilement observables dans le cas des vapeurs métalliques.

Si l'énergie des photons produits par DFM est inférieur à celle des photons créés par SFM, la condition d'accord de phase est par contre beaucoup moins contraignante pour le DFM que pour le SFM. La condition d'accord de phase résulte schématiquement du fait que (dans le cas du THG par exemple), le rayonnement triplé créé au niveau de chaque atome successif doit rester en phase avec le rayonnement excitateur sur toute la longueur du trajet optique compte tenu de la différence des indices de réfraction (ou de la dispersion) du milieu aux deux longueurs d'onde. La 
condition d'accord de phase dépend en outre de la longueur du milieu actif et de la géométrie du faisceau source (caractérisée dans le cas d'un faisceau gaussien par son paramètre confocal). Cette contrainte se traduit dans le cas du SFM (THG inclus), pour des faisceaux focalisés, par la condition sur les vecteurs d'onde

$$
\overrightarrow{\Delta \mathrm{k}}=\overrightarrow{\mathrm{k}}_{\mathrm{vuv}}-\left(\overrightarrow{\mathrm{k}}_{1}+\overrightarrow{\mathrm{k}}_{2}+\overrightarrow{\mathrm{k}}_{3}\right)<0 \text { ou } \overrightarrow{\Delta \mathrm{k}}=\overrightarrow{\mathrm{k}}_{\mathrm{vuv}}-3 \overrightarrow{\mathrm{k}}_{1}<0
$$

limitant ainsi la gamme de longueur d'onde de conversion à l'aile bleue des raies de résonance [6]. Cette limitation peut être partiellement levée par l'utilisation d'un mélange de gaz ayant des dispersions de signes opposés.

Afin d'améliorer le rendement de conversion de tels processus, il est courant de choisir les fréquences $\omega_{1}+\omega_{2}$ (ou $2 \omega_{1}$ ) fixes et en résonance avec un niveau de l'atome ou de la molécule (résonance à 2 photons). Dans ce cas, l'un des termes figurant au dénominateur de l'expression du $\chi^{(3)}$ tend vers zéro et le rendement de conversion peut augmenter d'un facteur $10^{3}$, tout en nécessitant une puissance de pompage du niveau résonant beaucoup plus faible. Cependant, la gamme d'accordabilité liée à la gamme de longueur d'onde couverte par le laser à la fréquence $\omega_{3}$ est plus courte, pour un colorant donné, que celle couverte par le triplement de fréquence non résonant. De plus, dans le cas des vapeurs métalliques peut s'ajouter l'effet de résonance supplémentaire dû à la présence de niveaux de Rydberg ou de niveau auto-ionisants qui contribuent à exalter la conversion. Il a été observé dans le mercure [7] que la présence d'une résonance à 4 photons pouvait être à l'origine d'un fort effet Stark permettant le THG, en géométrie focalisée, à des Iongueur d'onde où $\overrightarrow{\Delta \mathrm{k}}>0$.

Ainsi, typiquement, partant de lasers-sources pulsés de puissance crête $1 \mathrm{MW}(10$ $\mathrm{mJ}$ ) avec des durées d'impulsion de $10 \mathrm{~ns}$ et une finesse de $0.1 \mathrm{~cm}^{-1}$, on peut obtenir dans les gaz rares des taux de conversion de $10^{-7}$ (non résonant) à $10^{-3}$ (résonant) produisant un rayonnement de puissance crête $0.1 \mathrm{~W}$ à $1 \mathrm{KW}(1 \mathrm{~nJ}$ à $10 \mathrm{~nJ})$ soit $10^{9}$ à $10^{13}$ photons/pulse avec une finesse de raie de $0.5 \mathrm{~cm}^{-1}$ soit une résolution spectrale d'environ 150000 . Le rendement de conversion peut atteindre $10^{-2}$ dans le cas des vapeurs métalliques.

La diffusion Raman-Anti-Stokes est également un processus de conversion non linéaire d'ordre impair $2 n+1$ fréquemment utilisé [24], produisant dans l'hydrogène un rayonnement cohérent accordable aux fréquences $\omega_{n}=\omega_{1}+n * 4155 \mathrm{~cm}^{-1}$ (n étant l'ordre du processus anti-Stokes).

\section{Techniques annexes}

Il est à noter que, sans l'ajout d'un système dispersif, les rayonnements visibles et UV ne sont pas séparés du rayonnement VUV en sortie de la cellule de conversion. Une grande variété de dispositifs est utilisée par les expérimentateurs lorsque la séparation s'avère nécessaire, allant du simple prisme au monochromateur et aux miroirs dichroïques.

Une vérification du fonctionnement correct selon le processus choisi consiste à observer la variation de la puissance de VUV généré en fonction des puissances des lasers générateurs. Une mesure relative de la puissance est suffisante dans ce cas comme dans celui où l'on veut normaliser un signal d'intérêt au coup par coup pour s'affranchir des variations de cette puissance. La mesure absolue de la puissance VUV est rarement précise, qu'elle résulte de mesures directes ou indirectes. Dans le premier cas, on utilise soit une chaîne spectroscopique faisant intervenir un grand nombre d'éléments (optiques, monochromateur, détecteur) dont les caractéristiques sont connues sans grande précision, soit une simple photodiode dont le rendement est mal défini. Les mesures indirectes sont déduites de la mesure de l'ionisation créée dans un gaz (NO, Acétone...) lorsque son potentiel d'ionisation est inférieur à l'énergie du photon VUV (130 nm, ou 7,7 eV dans le cas de NO).

Une contrainte expérimentale supplémentaire est liée à la transparence des matériaux optiques. Elle trace une frontière nette entre les techniques utilisables de part et d'autre de $105 \mathrm{~nm}$, limite de transmission du fluorure de lithium $\mathrm{LiF}$, et délimite 
les domaines du VUV (105 nm $<\lambda<190 \mathrm{~nm})$ de celui de XUV $(\lambda<105 \mathrm{~nm})$. Dans le domaine du VUV, les trois éléments: cellules de conversion, d'interaction et de mesure de la puissance VUV sont physiquement séparés tandis que dans le domaine du XUV le système est ouvert, nécessitant entre chaque élément des zones intermédiaires de pompage différentiel. Dans ce dernier cas, afin de limiter la capacité de pompage utile, les montages expérimentaux font appel à des jets pulsés, tant pour le milieu étudié que pour le milieu de conversion. Par un choix judicieux de la distance entre l'orifice du jet et la zone d'interaction avec le laser, de la géométrie du jet et de sa densité, il est possible d'obtenir un fonctionnement stable de la conversion [8], utilisable à des fins spectroscopiques. Les systèmes à jets pulsés offrent, outre l'avantage de réduire les effets d'autoabsorption du rayonnement VUV généré, celui d'être beaucoup moins sensibles à la condition d'accord de phase puisque la zone d'interaction est plus courte qu'en cellule statique. D'autres dispositifs, plus sophistiqués qu'un simple trou ou qu'une fine feuille métallique percée par le laser lui-même, sont utilisés pour séparer les cellules, comme les substrats de galettes de microcanaux ou les capillaires qui canalisent sans l'absorber le rayonnement VUV.

La calibration absolue en longueur d'onde du VUV peut être d'une grande précision puisqu'elle est effectuée en calibrant les lasers-sources par référence à des standards dans le visible ou le proche UV. Deux techniques sont couramment utilisées dans ce but : l'effet optogalvanique dans une lampe à cathode creuse ( $\mathrm{Fe} / \mathrm{Ne}$ par exemple) et l'absorption par une cellule à Iode, Tellure ou Uranium [9]. Une précision absolue de $0.002 \mathrm{~cm}^{-1}$ peut être obtenue avec la cuve à Iode donnant une calibration théorique dans l'XUV de $0.012 \mathrm{~cm}^{-1}$.

Citons enfin la conversion de fréquence à des ordres supérieurs ou égaux à 5 [10]. Son intérêt pour une utilisation spectroscopique est, pour l'instant, relativement limité, du fait des très faibles puissances obtenues. L'étude du processus de conversion en soi motive la recherche d'harmoniques très élevés, la limite étant à l'heure actuelle l'harmonique 33 obtenu dans le Xénon à l'aide d'un laser Yag picoseconde de puissance supérieure à $10^{13} \mathrm{~W}$ [11].

\section{Les développements récents}

L'article de revue de Vidal [12] présente une étude détaillée des processus de conversion et de leur éventuelle compétition dans les vapeurs métalliques (notamment $\mathrm{Mg}$ et $\mathrm{Sr}$ ) et donne la liste exhaustive (en 1987) de tous les milieux de conversion et des processus associés. La conversion dans les gaz rares a été systématiquement étudiée par le groupe de Bielefeld [13] dans le cas non résonant [10] (110 à $129 \mathrm{~nm}$ par SFM et 160 à $207 \mathrm{~nm}$ par DFM dans le Xénon) et résonant [14] (72,5 à 83,5 par SFM et 127 à $180 \mathrm{~nm}$ par DFM dans le Krypton). Aux courtes longueurs d'onde, l'influence de processus concurrentiels REMPI se manifeste, caractérisée par des pics d'absorption dus à đes résonances avec des niveaux de $\mathrm{Kr}^{+}$.

Une utilisation systématique du SFM et du DFM résonant dans les gaz rares Xe et Kr utilisant les résonances à deux photons à $193.5 ; 202 ; 212.6 ; 216.6 \mathrm{~nm}$ pour le Krypton et 226; $255.6 \mathrm{~nm}$ pour le Xénon permet de couvrir pratiquement sans trou la gamme 72-210 nm grâce à l'emploi d'un laser à colorant pompé par Yag, de l'ensemble des Rhodamines (750-550 nm), des Coumarines (550-370 nm), du doublage (370-275 $\mathrm{nm}$ ) et du mixage après doublage $(275-220 \mathrm{~nm})$. Grâce à la résonance à 2 photons $4 \mathrm{p}^{5} 5 \mathrm{p}[1 / 2,0]$ à $212.55 \mathrm{~nm}$, on obtient dans le Krypton [15] un rendement de conversion de $5.10^{-4}$ avec des énergies de $110 \pm 60 \mathrm{~nJ}$ et accordable entre 121 et 201 $\mathrm{nm}$. L'optimisation de l'accord de phase par adjonction d'argon permet d'atteindre un rendement de $5.10^{-2}$ et $10 \mathrm{~mJ}$ (pulses de $20 \mathrm{~ns}$ ). La technique des jets pulsés pour les gaz rares est utilisée systématiquement en dessous de $105 \mathrm{~nm}$ par de nombreuses équipes [16][17][18][19][20].

La conversion dans les vapeurs métalliques a également été étudiée en détail dans $\mathrm{Mg}, \mathrm{Zn}$ et $\mathrm{Hg}$ de 105 à $175 \mathrm{~nm}$ [21]. Impliquant l'utilisation de fours de type "heat-pipe" doubles fonctionnant à des températures élevées $(\approx 1200 \mathrm{~K})[22][23]\left[23^{\prime}\right]$, les vapeurs 
métalliques $(\mathrm{Sr}, \mathrm{Zn}, \mathrm{Cd}, \mathrm{Mg}, \mathrm{Ca}$ ) ont tendance à être remplacées par le mercure d'un emploi relativement plus aisé [25][26][27][28][29][30]. Smith \& coll.[26] en particulier obtiennent en géométrie parallèle un rendement de conversion de $5 \%$ et une énergie de $1 \mathrm{~mJ}$ de 120 à $140 \mathrm{~nm}$ grâce à l'utilisation d'une quasi résonance à 1 photon suivie d'une résonance à 2 photons et d'une quasi résonance à 3 photons (à $130 \mathrm{~nm}$ ). L'impressionnante chaîne de lasers utilisée, même si elle préfigure les systèmes d'avenir n'en fait toutefois pas un modèle accessible à tous les laboratoires. Les trois longueurs d'onde sont obtenues par trois chaînes identiques comportant un laser à colorant pompé par un laser Yag doublé monomode et piloté par un laser à colorant en anneau injecté par un laser continu à argon ionisé. L'étage final d'amplification est constitué de cristaux de Saphir dopé au titane $\left(\mathrm{TiAl}_{2} \mathrm{O}_{3}\right)$ pompés par un laser Yag doublé fonctionnant en mode bloqué et fournissant des trains de $200 \mathrm{~ns}$ formés d'impulsions de $100 \mathrm{ps}$ à $10 \mathrm{~ns}$ d'intervalle. La finesse de raie est de $0,075 \mathrm{~cm}^{-1}$ soit une résolution de $10^{6}$.

L'utilisation de lasers Yag monomodes injectés pour le pompage des lasers à colorant permet d'augmenter la puissance crête et donc le rendement de conversion en réduisant la durée de l'impulsion tout en augmentant la finesse spectrale du laser.

Il est courant de travailler dans le VUV avec des finesses spectrales de l'ordre de 1 $\mathrm{cm}^{-1}$ qui offrent déjà une résolution spectrale comparable à celle des meilleurs spectrographes dans ce domaine de longueurs d'onde. L'utilisation d'étalons intracavité tant au niveau du Yag que des lasers à colorant permet de l'affiner sans difficulté à $0,1 \mathrm{~cm}^{-1}$. Ce résultat peut également être obtenu en utilisant une cavité de type Littman modifiée [31]. Grâce à l'emploi de lasers à colorant pulsés pilotés par laser continu, il est possible d'atteindre une très grande finesse spectrale $\Delta v$ proche de la limite de Fourier correspondant à la durée $\tau$ de l'impulsion laser $(\Delta v . \tau<0.7)$. Le groupe de Berkeley [18] obtient $210 \mathrm{MHz}$ soit $0.007 \mathrm{~cm}^{-1}$ ou une résolution de $1,510^{7}$, dans le XUV avec un jet pulsé de Xénon, dans la gamme 74 à $124 \mathrm{~nm}$, résultat proche de celui qu'avaient réalisé Cabaret \& coll.[32] à $121.6 \mathrm{~nm}$ et qui donne ainsi accès à la très haute résolution spectrale.

Le développement de lasers continus couvrant le domaine de l'XUV est en cours de réalisation [33]. Dans des vapeurs métalliques de $\mathrm{Sr}, \mathrm{Ca}, \mathrm{Mg}, \mathrm{Zn}$ et avec un four très simple, on obtient par SFM ou THG de 137 à $217 \mathrm{~nm}$ des puissances modestes de 0,5 pw à $60 \mathrm{nw}$ (soit $510^{5}$ à $610^{10}$ photons/s) principalement limitées par la puissance des lasers-sources (dye CW monomode pompé par laser à argon ionisé délivrant 0.2 à $3 \mathrm{~W}$ ).

Il apparaît ainsi que les développements récents des techniques de génération de rayonnement VUV et XUV cohérent ont porté sur tous les éléments du système, permettant ainsi le développement de techniques spectroscopiques de plus en plus élaborées.

\section{Utilisation des lasers VUV et XUV en physique atomique et moléculaire}

Grâce aux perfectionnements dont ils continuent à bénéficier, les lasers VUV et XUV produisent maintenant des flux de photons suffisants pour réaliser dans de bonnes conditions des études spectroscopiques par les techniques autrefois réservées aux seuls lasers visibles et UV. Ils offrent des possibilités spécifiques pour l'étude de nombreux problèmes de physicochimie fondamentale ou appliquée que les techniques traditionnelles ne permettent pas d'atteindre, sinon au prix de difficultés supplémentaires. En particulier, l'excitation directe à un photon VUV de niveaux énergétiquement élevés présente l'avantage, par rapport aux techniques multiphotoniques précédemment employées, de ne pas nécessiter les flux élevés de photons qui sont la cause de problèmes annexes parasites (voies d'excitations secondaires, déplacement et élargissement des niveaux par divers effets dus aux champs électriques intenses). De plus, compte tenu des règles de sélection, le VUV permet d'accéder, à un photon, à des niveaux différents de ceux autorisés par une excitation multiphotonique, tout en bénéficiant de sections efficaces d'absorption de 4 à 8 ordres de grandeur supérieures à celles à deux ou trois photons. Enfin, outre 
l'utilisation comme source primaire de rayonnement, la génération non linéaire peut être utilisée comme outil de diagnostic du milieu dans lequel elle se produit avec une extrême sensibilité compte tenu des caractéristiques de cohérence et de directivité du rayonnement résultant [34].

Depuis la revue publiée par Rostas [35] en 1986, les études fondées sur l'utilisation du VUV ont gagné en sophistication, couplant de façon très complète les méthodes et techniques de base. Elles seront présentées, sans prétendre être exhaustif, ainsi que les espèces auxquelles elles ont été appliquées .

Les utilisations fondamentales portent sur la physique des lasers et le développement des sources cohérentes dans le VUV, la spectroscopie à haute et très haute résolution des atomes et des molécules, la physique moléculaire sous l'aspect photophysique (photodissociation et photoionisation) avec la caractérisation des fragments produits et de leur état d'énergie interne et sous l'aspect photodynamique en relation avec les calculs ab initio de surfaces de potentiel (et leurs éventuelles intersections) pour expliciter les voies réactionnelles vers les produits finaux et leurs caractéristiques.

L'action d'un rayonnement sur un milieu se traduit finalement par trois phénomènes fondamentaux: l'absorption, la fluorescence induite, l'ionisation (mono ou multiphotonique) ainsi que d'éventuels processus secondaires (non radiatifs ou collisionnels). Les méthodes de base utilisées seules, ou plus souvent en combinaison, peuvent être regroupées schématiquement sous les rubriques suivantes:

a) La spectroscopie d'excitation à un photon VUV accordable avec détection de l'absorption [36] ou de la fluorescence totale qui permet de mettre en évidence, si le spectre d'excitation ne coïncide pas avec le spectre d'absorption, l'existence de perturbations, prédissociations ou autoionisations. Dans le cas de plusieurs systèmes électroniques se recouvrant, la détection de la fluorescence peut être faite avec sélection de fréquence.

b) La spectroscopie de fluorescence à un photon VUV de longueur d'onde fixe et détection de la fluorescence résolue en longueur d'onde, méthode appropriée à la mesure des facteurs de Franck-Condon et de Hönl-London. La spectroscopie dite d'émission Raman résonante est une extension récente de cette méthode [37][38]. Le spectre d'émission Raman permet d'étudier (avec une grande résolution temporelle même en utilisant des lasers nanoseconde) la dynamique de la dissociation et apporte des informations sur la structure et la géométrie des niveaux liés et des états dissociatifs ainsi que sur les mécanismes de prédissociation mis en jeu.

c) La spectroscopie résolue en temps permet d'observer l'influence de perturbations éventuelles entre niveaux singulets et triplets [22], dans le cas où plusieurs niveaux interviennent avec des durées de vie différentes. Une nouvelle voie développée par le groupe de Zewail [39], encore peu explorée dans le VUV, est ouverte par l'utilisation de lasers femtoseconde. Elle s'intéresse à la dynamique d'évolution de la réaction vers les produits finaux à une échelle de temps suffisamment faible pour observer les oscillations entre les différents états mis en jeu. Cette méthode complète les résultats obtenus par spectroscopie d'émission Raman.

d) La spectroscopie de photodissociation (ou de photofragmentation) avec détection et analyse sélective des produits de réaction est un moyen d'accès très efficace aux surfaces de potentiel et à la dynamique des systèmes moléculaires. Elle s'intéresse aux rapports de branchement entre les divers voies de dissociation, dans le cas de molécules polyatomiques (jusqu'aux agrégats et aux clusters [23]) et permet de mettre à l'épreuve les calculs théoriques. Le mécanisme de la photodissociation (directe ou par prédissociation) est souvent désigné sous le concept de demi-collision: la photodissociation ne représente en effet que la seconde partie d'une collision bimoléculaire complète puisque la molécule est préparée instantanément dans un état excité par absorption d'un photon, sans changement de géométrie. Les travaux portent sur l'analyse des grandeurs scalaires et vectorielles associées aux fragments (atomes, radicaux, ions ou photoélectrons): énergie interne et distribution d'énergie translationnelle, corrélations vectorielles entre les différents moments et vecteurs mis 
en jeu, distributions angulaires, ainsi que l'étude des profils Doppler obtenus dans différentes géométries de lasers pompe et sonde et qui reflètent les mouvements des fragments [40][41]. La technique Vads (velocity aligned Doppler spectroscopy) [42] est un exemple d'emploi de cette méthode désignée aussi sous le nom de spectroscopie de translation. La fluorescence induite par laser est également un moyen de sonder les fragments rovibrationnellement excités au fondamental.

e) La spectroscopie d'excitation à deux étapes par OODR (optical optical double resonance) résulte de la préparation par un photon VUV puis d'un photon UV ou visible d'un niveau bien déterminé. La méthode permet l'analyse des perturbations, prédissociations, transitions induites par collisions, structures fines ... Elle est bien illustrée par les travaux du groupe Vidal [22]. Elle est appliquée, entre autres, à l'étude des interactions entre états de Rydberg qui entraînent prédissociation et/ou autoionisation. Avant l'utilisation des lasers VUV la première étape pouvait correspondre à une transition à 2 ou 3 photons. Cette méthode est couplée à la suivante lorsque la dernière étape (ou un troisième photon) conduit à l'ionisation.

f) La spectroscopie d'ionisation dite MPI à ionisation photonique simple ou REMPI (resonantly enhanced multiphoton ionization), plus sensible, résulte de l'action d'un photon de pompe VUV résonant noté 1 et d'un photon d'ionisation noté 1', d'où la dénomination REMPI $\left(1+1^{\prime}\right)$. Cette méthode étudie les fragments (ions ou photoélectrons) à l'aide des techniques usuelles de spectrométrie de masse (quadrupolaire ou à temps de vol), avec détection des coïncidences, des seuils d'apparition. La PES (photoelectron spectroscopy) est fondée sur la mesure des énergies cinétiques et des distributions angulaires des électrons produits. Son développement ZEKE-PES (zero kinetic energy) (dans lequel les électrons de seuil sont extraits par un champ électrique retardé et qui offre une résolution de l'ordre de quelques $\mathrm{cm}^{-1}$ comparés aux 5 à $10 \mathrm{meV}$ de la méthode PES) est l'objet de nombreux travaux [43][19]. Compte tenu de sa complémentarité avec la méthode REMPI $\left(1+1{ }^{\prime}\right)$, quelques travaux faisant appel au REMPI (2+1') (2 désigne ici une transition à 2 photons, en général UV, obtenus par doublage dans un cristal au voisinage de $200 \mathrm{~nm}$ ) sont référencés ici [44][45][46][47][48][49]. Cette méthode est enfin très souvent associée à l'OODR dans les techniques REMPI $\left(1+1^{\prime}+1^{\prime \prime}\right)$. C'est la méthode de choix pour l'étude des niveaux de Rydberg élevés et des niveaux autoionisants à proximité du seuil d'ionisation.

Les applications récentes des lasers VUV portent sur a) La spectroscopie à haute résolution concernant a1) les structures moléculaires d'états excimères des gaz rares [50][51][52], d'états de Rydberg autoionisés de $\mathrm{H}_{2}$ [53][54], $\mathrm{NO}$ [55], $\mathrm{HCl}, \mathrm{HBr}$ [56], d' interactions entre états de Rydberg et états de valence de $\mathrm{N}_{2}$ [19][57], $\mathrm{O}_{2}, \mathrm{CO}_{2}$ [19], $\mathrm{HCl}$ [58], de complexes de Van der Waals de ArNO [49], d'états doublement excités de Ba [59], et de l'effet Stark sur les états de Rydberg de $\mathrm{H}_{2}$ [60], HI [61]. a 2) la photoionisation d'états de Rydberg de $\mathrm{H}_{2} \mathrm{O}$ [17], la formation de paires d'ions et leurs interactions $\mathrm{I}_{2}, \mathrm{ICl}[62], \mathrm{Cl}_{2}$ [51]. a3) les mesures de haute précision d'énergie d'ionisation $\mathrm{H}_{2}$ [63][64], de niveaux de Rydberg, de structure isotopique $\mathrm{CO}, \mathrm{N}_{2}$ [65], de structure fine $\mathrm{H}$ [32], $\mathrm{Kr}$ [66]. b) La dynamique moléculaire $\mathrm{H}_{2} \mathrm{O}$ [67], avec b1) l'énergie interne des fragments, les transferts d'énergie translation-rovibration $\mathrm{CO}$ [28][68][69][70], OH[69], b2) l'énergie cinétique des fragments et les corrélations vectorielles OCS [23], $\mathrm{NO}$ [24], $\mathrm{CO}_{2}$ [70], b3) l'émission Raman $\mathrm{H}_{2} \mathrm{O}, \mathrm{C}_{2} \mathrm{H}_{4}, \mathrm{H}_{2} \mathrm{~S}$ [37], b4) La photodissociation et l'analyse des prédissociations $\mathrm{CO}$ [65][22], $\mathrm{H}_{2} \mathrm{O}$ [67][71], $\mathrm{H}_{2}$ [46]. c) la mesure des durées de vie $\mathrm{CO}$ [30] $\mathrm{CO}, \mathrm{N}_{2}$ [65], $\mathrm{N}_{2}$ [19]. d) La détection d'atomes et de molécules à de très faibles concentrations $\mathrm{O}, \mathrm{N}, \mathrm{H}$ [34], $\mathrm{N}_{2}$ [47], $\mathrm{CO}$ [68] et des espèces métastables $\mathrm{NO}^{*}$ [72].

La conversion non linéaire dans les gaz moléculaires, grâce à la présence de résonance fournissant les zones de dispersion anormale propices au mélange de fréquence, est un puissant outil d'analyse spectroscopique de ces milieux. Le flux de photons VUV est en général plus faible que dans les gaz rares, sauf en cas de résonance à deux photons [73][74][75][76][77][78][79]. 
Les utilisations appliquées, sans que la frontière avec les utilisations fondamentales soit toujours nette, concernent les diagnostics sur les plasmas de fusion [25][45][54][80], la photochimie comme la pyrolyse et la caractérisation des produits de combustion dans les flammes [81][82] (cette dernière référence utilisant la combinaison originale du VUV et de l'ionisation par les électrons créés par le laser sur un filament métallique), le traitement des surfaces (par exemple le rôle de l'hydrogène en surface dans le dépôt de carbone diamant [83]), la lithographie etc... L'étude de la photochimie de la désorption des molécules en surface est un champ en pleine expansion, s'intéressant à une grande variété de processus: photodissociation adjacente ou sur la surface, dissociation multiphotonique des absorbats, combinaison d'effets photolytiques et pyrolytiques, chimisorption dissociative et à l'inverse désorption associative [84][85][86] [83][87] [88][89][90][91]. Citons enfin la photolyse de molécules atmosphériques [92] ou participant à la pollution atmosphérique [93].

\section{BIBLIOGRAPHIE}

* désignent les articles de revue

[ 1] Reintjes J.F, Coherent UV andVUV source in Laser Handbook, Elsevier Science (1985)

[2] New G.H.C. Ward J.F. Phys. Rev. Lett. 19 (1967) 556

Ward J.F., New G.H.C. Phys. Rev. 185 (1969) 57

[ 3] Harris S.E., Miles R.B. Appl. Phys. Lett. 19 (1971) 385

Kung A.H., Young J.F., Bjorklund G.C., Harris S.E. Phys. Rev. Lett. 29 (1972) 985

Miles R.B., Harris S.E. IEEE J. Quant. Elec. 9 (1973) 470

[4] Hanna DC Yuratich MA Cotter D Nonlinear optics of free atoms and molecules, Springer Verlag, (1979)

Butcher P.N., Cotter D. The elements of non linear optics, Cambridge University Press (1990)

[5] Kato K IEEE J. Quant. Elec. 26 (1990) 1173

Chen C.IT. Opto 64 (1991) 40

Borsutzky A., Brünger R., Huang Ch, Wallenstein R. Appl. Phys. B. 52 (1991) 55

Watanabe M., Hayasaka K., Imajo H., Umezu J., Urabe S. Appl. Phys. B. 53 (1991) 11

Wu B., Xie F., Chen C.T., Deng D., Xu Z. Opt. Commun. 88 (1992) 451

[ 6] Mahon R., Mc Ilrath T.J., Myerscough V.P., Koopman D.W. IEEE J. Q. Elec. 15 (1979) 444

[7] Efthimiopoulos T., Koudoumas E. Appl. Phys. B.,54 (1992) 193

Payne M.G., Miller J.C., Hart R.C., Garett W.R. Phys. Rev A 44 (1991) 7684

[ 8] Rettner C.T., Marinero E.E., Zare R.N., Kung A.H. J. Phys. Chem. 88 (1984) 4459

[ 9$]$ Nave G., Learner R.C.M., Thorne A.P., Harris C.J. Josa: B 8 (1991) 2028

Gagné J.M., Babin F. J. Phys. II France 2 (1992) 827

[10] Hilbig R., Wallenstein R. Appl. Opt. 21 (1982) 913

[11] Lompré LA., L'Huillier A., Ferray M., Manot P., Mainfray G., Manus C. Josa B. 7 (1990) 754 Kulander K.C., Shore B.W. Phys. Rev. Lett 62 (1989) 524 \& Josa B 7 (1990) 502

L'Huillier A., Lompré L.A., Mainfray G., Manus C., in Proc. Sth. Int. Conf. on multiphoton Processes (Paris), (1991) 45

Miyazaki K., Sakai H. J. Phys. B. 25 (1992) L83

[12] Vidal C.R., Four wave mixing in Tunable Lasers, Eds. Mollenhauer, White Springer (1987) 57

[13] Hilbig R., Hilber G., Lago A., Wollf B., Watlenstein R. Comm. At. Mol. Phys. 4 (1986) 157

[14] Hilber G., Lago A., Wallenstein R. Josa B. 4 (1987) 1753

[15] Marangos J.P., Shen N. Ha H., Hutchinson MHR., Connerade J.P. Josa B. 7 (1990) 1254

[16] Bethune D.S., Rettner C.T. IEEE J. Quant. Elec. 23 (1987) 1348

[17] Page R.H., Larkin R.J., Kung AH., Shen Y.R., Lee Y.T. Rev. Sci. Instr. 58 (1987) 1616 Page R.H., Larkin R.J., Kung AH., Shen Y.R., Lee Y.T. J. Chem. Phys. 88 (1988) 2249

[18] Cromwell E.F., Kung A.H., Trickl T., Lee Y.T. Proc. SPIE 912 (1988) 145

Proch D., Trickl T. Rev. Sci. Instr. 60 (1989) 713

Cromwell E.F., Trickl T., Lee Y.T., Kung A.H. Rev. Sci. Instr. 60 (1989) 2888

Trickl T., Cromwell E.F., Lee Y.T., Kung A.H. J. Chem. Phys. 91 (1989) 6006

[19] Softley T.P., Ernst W.E., Tashiro L.M., Zare R.N. Chem. Phys. 116 (1987) 299

Ubachs W., Tashiro L.M., Zare R.N. Chem. Phys. 130 (1989) 1

Fielding H.H., Softley T.P., Merkt F. Chem. Phys. 155 (1991) 257

[20] Tonkyn R.G., White M.G. Rev. Sci. Instr. 60 (1989) 1245

Tonkyn R.G., Winniczek J.N., White M.G. Chem. Phys. 164 (1989) 137

[21] Herman PR La Rocque PE Lipson RH Jamroz W Stoïcheff BP Can J. Phys. 63 (1985) 1581 Herman P.R., La Rocque P.E., Stö̈cheff B.P. Josa B. 10 (1985) 502

[22] Klopotek P., Vidal C.R. Can. J. Phys. 62 (1984) 1426

Scheingraber H., Vidal C.R. Josa B 2 (1985) 343

Strobl K., Vidal C.R. J. Chem. Phys. 86 (1987) 62 
[23] Sivakumar N., Hall G.E., Houston P.L., Hepburn J.W. J. Chem. Phys. 88 (1988) 3692 Strauss CE McBane GC Houston PL Burak I Hepburn JW J. Chem. Phys. 95 (1991) 5364

[23'] Zhao S.Y., Zhang P.L., Zhang G.Z., Zhao W.Z. Appl. Optics 28 (1989) 4521

[24] Allendorf S.W., Leahy D.J., Jacobs D.C., Zare R.N. J. Chem. Phys. 91 (1989) 2216 Leahy J.D., Reid K.L., Zare R.N. J. Chem. Phys. 95 (1991) 1757

[25] Stutzin GC Young AT Schlachter AS Stearns JW Leung KN Kunkel DW Worth GT Stevens R Rev. Sci. Instr. 59 (1988) 1363; Stutzin Young Schlachter Leung Kunkel Chem. Phys.Lett. 155(1989)475; Stutzin Young Döbele Schlachter Leung Kunkel Rev Sci Instr 61(1990)619 Lemaire J.L., Rostas F. (rapports internes)

[26] Smith A.V., Hadley G.R., Esherick P., Alford W.J. Josa B. 9 (1987) 708 Smith A.V., Alford W.J. Josa B. 11 (1987) 1765 Smith A.V., Alford W.J. Josa B. 7 (1988) 1503

[27] Muller III CH Lowenthal DD DeFaccio MA Cimolino M Hauck JF Proc. SPIE 912(1988)106 Muller III C.H., Lowenthal D.D., DeFaccio M.A., Smith A.V. Josa B. 13 (1988) 651

[28] Rice J.K., Baronavski A.P. J. Chem. Phys. 94 (1991) 1006

[29] Turley RS McFarlane RA Remillard J Steel DG Proc. SPIE 912(1988)116

[30] Hallstadius H., Lundberg H.Phys. Scripta 40 (1989) 652

[31] Hart D.J., Hepburn J.W. Chem. Phys. 129 (1989) 51 Hart D.J., Bourne O.L.,Chem. Phys. 133 (1989) 103

[32] Cabaret L., Delsart C., Blondel C. Opt. Commun. 61 (1987) 116

[33] Nolting J., Kunze H., Schütz I., Wallenstein R. Appl. Phys.B 50 (1990) 331

[34] Celii F.G., Thorsheim H.R., Hanratty M.A., Butler J.E. Appl. Opt. 29 (1990) 3135 Celii F.G., Thorsheim H.R., Butler J.E., Plano L.S., Pinneo J.E. J. Appl. Phys. 68 (1990) 5237 Celii F.G., Thorsheim H.R., Butler J.E. J. Chem. Phys. 94 (1991) 5248

[35] Rostas F. Annales de Physique Coll n 3 Supplt 311 (1986) 39

[36] Yang X.F., Lemaire J.L., Rostas F., Rostas J. Chem. Phys. Lett. (accepté, sous presse)

[37] Sension R.J., Brudzynski R.J., Hudson B.S. Phys. Rev. Lett. 61 (1988) 694 Sension R.J.,Hudson B.S. J. Chem. Phys. 90 (1989) 1377 Sension R.J., Brudzynski R.J., Hudson B.S., Zhang J., Imre D.G. Chem Phys. 141 (1990) 393 Brudzynski R.J.,Sension R.J., Hudson B.S. Chem Phys. Lett. 165 (1990) 487

[38] Henriksen N.E., Zhang J., Imre D.G. J. Chem Phys. 89 (1988) 5607 Zhang J., Imre D.G. J. Chem Phys. 90 (1989) 1666 Lee S.Y., Freed K.F. J. Chem. Phys. 90 (1989) 7030 Jacon M., Atabek O., Leforestier C. J. Chem Phys. 91 (1989) 1585

[39]*Metiu H., Engel V. Josa B 7 (1990) 1709

Khundkar L.R., Zewail A.H. Ann. Rev. Phys. Chem. 41 (1990) 15

Dantus M., Roberts G. Comm. Atom. Molec. Phys. 26 (1991) 131

Dantus M., Janssen M.H.M., Zewail A.H. Chem. Phys. Lett. 181 (1991) 281

[40] Biesner J Schnieder L Ahlers G Xie X Welge KH Ashfold MNR Dixon RN JCP 91(89)2901 Schnieder L Meier W Welge KH Ashfold MNR Western CM J. Chem. Phys. 92 (1990) 7027 Segall J., Wen Y., Lavi R., Singer R., Wittig C. J. Phys. Chem. 95 (1991) 8078 Ashfold M.N.R., Schnieder L., Welge K.H. Faraday Disc. Chem. Soc. 91 (1991) 128 Shafer N., Bersohn R. J. Chem. Phys. 94 (1991) 4817 Stolow N Balko BA Cromwell EF Zhang J Lee YJ J. Photochem. Photobiol. 62 (1992) 285 *Ashfold MNR Lambert IR Mordaunt DH Morley GP Western CM J Phys Chem 96(1992)2938

[41] Brouard M Martinez MT Milne CJ Simons JP Wang JX Chem. Phys. Lett. 165 (1990) 423

[42] Xu Z., Koplitz B., Wittig C. J. Chem. Phys. 87 (1987) 1062 Xu Z., Koplitz B., Wittig C. J. Chem. Phys. 90 (1989) 2692

Xie X Schnieder Wallmeier H Boettner R Welge KH Ashfold MNR JCP 92 (1990) 1608 Continetti R.E., Balko B.A., Lee Y.T. Chem. Phys. Lett. 182 (1991) 400

[43] Reiser G., Habenicht W., Müller-Dethlefs K., Schlag E.W. Chem. Phys. Lett. 152 (1988) 119 Habenicht W., Reiser G., Müller-Dethlefs K., J. Chem. Phys. 95 (1991) 4809

*Müller-Dethlefs K., J. Chem. Phys. 95 (1991) 4821

Reiser G., Müller-Dethlefs K., J. Phys. Chem. 96 (1992) 9

Rieger D., Reiser G., Müller-Dethlefs K., Schlag E.W. J. Phys. Chem. 96 (1992) 12

[44] Gadd G.E., Huestis D.L., Slanger T.G. J. Chem. Phys. 95 (1991) 3944

[45] Huo WM Rinnen KD Zare RN J. Chem. Phys. 95 (1991) 205 Rinnen KD Buntime MA Kliner DAV Zare RN Huo WM J. Chem. Phys. 95 (1991) 214 Kliner D.A.V., Adelman D.E., Zare R.N. J. Chem. Phys. 94 (1991) 1069

[46] Buntime M.A., Baldwin D.P., Chandler D.W. J. Chem. Phys. 96 (1992) 5843

[47] Lykke K.R., Kay B.D. J. Chem. Phys. 95 (1991) 2252

[48] Ondrey G.S., Rose C., Proch D., Kompa K.L. J. Chem. Phys. 95 (1991) 7823

[49] Takahashi M. J. Chem. Phys. 96 (1992) 2594

[50] Tsukiyama K., Kasuya T. J. Molec. Spectr. 151 (1992) 312

[51] Tsuchizawa T., Yamanouchi K., Tsuchiya S. J. Chem. Phys. 93 (1990) 112 Tsuchizawa T., Yamanouchi K., Tsuchiya S. J. Chem. Phys. 92 (1990) 1560

[52] Pratt S.T., Dehmer P.M., Dehmer J.L. Chem. Phys. Lett. 165 (1990) 131 
[53] Pratt S.T., McCormack E.F., Dehmer J.L., Dehmer P.M. Phys. Rev. Lett. 68 (1992) 584 O'Halloran MA Dehmer PM Tomkins FS Pratt ST Dehmer JL J. Chem. Phys. 89 (1988) Dehmer JL Dehmer PM Pratt ST Tomkins FS O'Halloran MA J. Chem. Phys. 90 (1989) 6243

[54] Kung A.H., Trickl T.,Gershenfeld N.A.,Lee Y.T. Chem. Phys.Lett. 144 (1988) 427 Meier W., Zacharias H., Welge K.H. Chem. Phys. Lett. 163 (1989) 88 Vershurr J.W.J., Vanden Heuvell V.L., Chem. Phys. 129 (1989) 1 Banerjee S. Chakrabati M.K., Bhattacharyya S.S., Saha S. J. Chem. Phys.95 (1991) 1608

[55] Fujii A., Ebata T., Ito M., J. Chem. Phys. 90 (1989) 6993

[56] Callaghan R., Huang Y.L., Arepalli S., Gordon R.J. Chem. Phys. Lett. 158(1989)531

[57] McCormack EF Pratt ST Dehmer PM Dehmer JLPhys Rev. A 45 (1992) 4697

[58] Green D.S., Bickel G.A., Wallace S.C. J. Molec. Spectr. 150 (1991) 303 Green D.S., Wallace S.C. J. Chem. Phys. 96 (1992) 5857 Xie Y., Reilly P.T.A., Chilikuri S., Gordon R.J. J. Chem. Phys. 95 (1991) 854

[59] Connerade J.P. J. Phys. B 24 (1991) L331

*Connerade J.P. J. Phys. II France 2 (1992) 757

[60] Fielding H.H., Softley T.P. Chem. Phys. Lett. 185 (1991) 199

[61] Mank A Drescher $M$ Huth-Fehre T Schönhense $G$ Böwering $N$ Heinzmann U J.Phys. B 22(1989)L487; Huth-Fehre Mank Drescher Böwering Heinzmann Phys. Rev. Lett. 64(1990)396; Mank Drescher Huth-Fehre Böwering Heinzmann Lefèbvre-Brion H J. Chem. Phys. B 95 (1991) 1676

[62] Lipson R.H., Hoy A.R. J. Chem. Phys.90 (1989) 6821 Hoy A.R., Lipson R.H. Chem. Phys. 140 (1990) 187

[63] McCormack E., Gilligan J.M., Eyler E.E. Phys. Rev. A 39 (1989) 2260

[64] Eyler E.E. Proc. SPIE 912 (1988) 132

[65] Levelt P.F., Ubachs W., Hogervorst W. J. Phys. II France 2 (1992) 801 Levelt P.F., Ubachs W. Chem. Phys. (à paraître)

[66] Hart D.J., Bourne O.L., Rayner D.M. Opt. commun. 73 (1989) 213

[67]*Engel V Stammler V Vander Wal RL, Crim FF Sension RJ Hudson B Andresen P Hennig S Weide K Schinke R J. Phys. Chem. 96 (1992) 3201

[68] Price JM Ludviksson A Nooney M Xu M Martin RM Woodtke AM JCP 96 (1992) 1854

[69] Gericke K.H., Göltzenleuchter H., Comes F.J.,Chem. Phys. 127 (1988) 379

Butenhof T.J., Carleton K.L., Moore C.B. J. Chem. Phys. 92 (1990) 377

Gericke K.H., Glaser H.G., Maul C., Comes F.J. J. Chem. Phys. 92 (1990) 411

[70] Miller R.L., Kable S.H., Houston P.L. J. Burak I. Chem. Phys. 96 (1992) 332 Wu M., Taylor D.P., Johnson P.M., J. Chem. Phys. 95 (1991) 761

[71] Shafizadeh N Rostas J Lemaire JL Rostas F Chem. Phys. Lett. 152(1988)75; Briggs R Halpern JB Hancock G Shafizadeh N Rostas J Lemaire JL Rostas F Chem. Phys. Lett. 152 (1988) 75

[72] Feigerle C.S., Miller J.C. J. Chem. Phys. 90 (1990) 2900

[73] Aguillon F., Lebéhot A., Rousseau J., Campargue R. J. Chem. Phys. 86 (1987) 5426 Faucher O., Aguillon F., Campargue R. J. Chem. Phys. 94 (1991) 4141

[74] Ashfold M.N.R., Prince J.D. Molec. Phys. 73 (1991) 297

[75] Merkt F., Softley T.P. Chem. Phys. Lett. 165 (1990) 477 Merkt F., Softley T.P. J. Chem. Phys. 92 (1990) 6426 Merkt F., Softley T.P. J. Chem. Phys. 93 (1990) 1540

[76] Tsukiyama K., Tsukakoshi M., Kasuya T. Appl. Phys. B 50 (1990) 23 Tsukiyama K., Tsukakoshi M., Kasuya T. J. Chem. Phys. 94 (1991) 883

[77] Czarnetzki U., Böbele H.F. Phys. Rev. A 44 (1991) 7530

[78] Wallace S.C., Ines K.K. J. Chem. Phys. 72 (1980) 4805

[79] Strauss C.E.M., Funk D.J. Opt. Letters 16 (1991) 1192

180] Pasch E., Bogen P., Mertens Ph. J. Nuclear Mat. 166,167 (1990) 455

[81] Tjossem P.J.H., Smyth K.C., J. Chem. Phys. 91 (1989) 2041

[82] Boyle JG Pfefferle LD Gulicicek EE Colson SD Rev. Sci. Instr. 62 (1991) 323

[83] Celii F.G., Butler J.E. Appl. Phys. Lett. 54 (1989) 11 Celii F.G., Nelson H.H., Pehrsson P.E. J. Mater. Res. 5 (1990) 2337

[84] Mase K., Mizuno S., Achiba Y., Murata Y. Surf. Sci. 242 (1991) 444 Mase K., Fukutani K., Murata Y. J. Chem. Phys. 96 (1992) 5523

[85] Mödl A., Robota H., Segner J., Vielhaber W. Lin M.C. Ertl G. J. Chem. Phys. 83(1985)4800

[86] Hanisco T.F., Yan C., Kummel A.C. J. Phys. Chem. 96 (1992) 2982

[87] Hasselbrink E Jakubith S Nettesheim S Wolf M Cassuto A Ertl G. JCP 92 (1990) 3154

[88] Messina M., Coalson R.D. J. Chem. Phys. 95 (1991) 8977

[89] Bourdon EBD Cho CC Das P Polanyi JC Stanners CD Xu GQ J. Chem. Phys. 95 (1991) 136

[90] Kolasinski K.W., Shane S.F.,Zare R.N. J. Chem. Phys. 95 (1991) 5482

[91] Sheng J., Zhang J.Z.H. J. Chem. Phys.96 (1992) 3866

[92] Gibson ST Lewis BR Baldwin KGH Carver JH Josa B 6(1989)1200 \& JCP 94 (1991) 1060

[93] Nourbakhsh S Norwood K Yin HM Liao CL Ng CY J. Chem. Phys.95 (1991) 946 \& 5014 